\title{
Embedding Theorems \\ for Sobolev-Besicovitch Spaces of Almost Periodic Functions
}

\author{
R. Iannacci, A. M. Bersani, G. Dell'Acqua and P. Santucci
}

\begin{abstract}
We show embedding theorems of Sobolev type for Sobolev-Besicovitch spaces $H_{a p}^{m, q}$ of almost periodic functions for any $q>1$ and, consequently, for spaces $W_{a p}^{m, q}$, with $q \in(1,2]$. The fundamental tool for the proof of the main theorem is the Hausdorff-Young theorem for almost periodic functions.
\end{abstract}

Keywords: Almost periodic functions, embedding theorems, Sobolev-Besicovitch spaces AMS subject classification: 42 A 75

\section{Introduction}

The theory of Sobolev-Besicovitch spaces $W_{a p}^{m, q}\left(\mathbb{R}^{s}\right)$ and $H_{a p}^{m, q}\left(\mathbb{R}^{s}\right)$ of almost periodic functions, already introduced by Pankov [11] for the case $q=2$ and by Avantaggiati $[5,6]$ for any $q \geq 1$, has been recently approached in the one-dimensional case by Avantaggiati, Bruno and Iannacci [3], with the aim of solving partial differential equations with almost periodic coefficients. In [3] the authors prove also some preliminary regularity results for $B_{a p}^{q}$-functions, and for as much as we know this is the only result obtained regarding this subject.

The main goal of this work is to find embedding theorems for Sobolev-Besicovitch spaces of such type. The $W_{a p}^{m, q}$ spaces, whose elements have countable spectrum, are wide spaces (they are not separable); so we restrict ourselves to study suitable subspaces where we fix the structure of the spectrum $\Lambda$, i.e. we suppose that it has only one limit point, the point at infinity, and the frequencies satisfy the fundamental relation

$$
\sum_{j=1}^{\infty} \frac{1}{\left|\lambda^{j}\right|^{\gamma}}<+\infty \quad \text { for all } \gamma>\beta \geq 0
$$

where $\beta$ is a suitable exponent that plays a fundamental role in the regularity of the embeddings. These subspaces are separable. In such spaces, the embeddings are refined

R. Iannacci: Univ. "La Sapienza", Dip. Met. Mod. Mat., via A. Scarpa 16, I - 00161 Roma A. M. Bersani: Univ. "La Sapienza", Dip. Met. Mod. Mat., via A. Scarpa 16, I-00161 Roma G. Dell'Acqua: Univ. "La Sapienza", Dip. Met. Mod. Mat., via A. Scarpa 16, I-00161 Roma P. Santucci: Univ. "La Sapienza", Dip. Met. Mod. Mat., via A. Scarpa 16, I-00161 Roma 
through Sobolev-type theorems, that coincide with the classical ones if we consider periodic functions, being in this case $\beta$ equal to the dimension of the space where they are defined.

While for any $q \in[1,2]$ the embedding theorems are proved (see also [9]), we have not found, at the moment, a way to extend our results for $q>2$. Therefore, using the Hausdorff-Young theorem for $B_{a p}^{q}$-functions, we first prove embedding properties between $W_{a p}^{m, q}$ and $H_{a p}^{m, q}$ spaces. Let us note that such embeddings are natural for their similarity to the analogous ones between classical $W^{m, q}$ and $H^{m, q}$ spaces. Then, we prove the embedding theorems for the $H_{a p}^{m, q}$ spaces, for any $q>1$. In this way, the corresponding theorems for the $W_{a p}^{m, q}$ spaces $(q \in(1,2])$ become a corollary, being in this case

$$
W_{a p}^{m, q} \hookrightarrow H_{a p}^{m, q} .
$$

The Hausdorff-Young theorem again plays a central and fundamental role in the proof of the theorems. When $q \geq 2$, we believe that there are good possibilities for finding contiguity properties for these spaces, therefore extending our results to any $\ddot{q}>1$.

In Section 2 we recall the definition of the spaces $B_{a p}^{q}$ and their main properties (in particular, the Hausdorff-Young theorem). In Section 3 we introduce the $W_{a p}^{m, q}$ spaces, defining strong derivatives of $B_{a p}^{q}$-functions, and we study the spaces $H_{a p}^{m, q}$ and their basic properties. In Section 4 we explore the relations between $W_{a p}^{m, q}$ and $H_{a p}^{m, q}$ spaces, showing the importance of the latter. In Section 5 we prove Sobolev embedding theorems for the spaces $H_{a p}^{m, q}$ for all $q>1$ (and, consequently, for $W_{a p}^{m, q}$, when $q \in(1,2]$ ). Finally, in Section 6 we underline the crucial role played by the parameter $\beta$, studying the case in which $\Lambda$ is an additive semigroup with a finite number $n$ of generators. In this case we find that $\beta=n$.

\section{Notations and definitions}

For any $s \in \mathbb{N}$, let $\mathcal{P}\left(\mathbb{R}^{s}\right)$ denote the complex vector space of all trigonometric polynomials of $s$ variables, that is $P \in \mathcal{P}\left(\mathbb{R}^{s}\right)$ if and only if there exist $c_{1}, \ldots, c_{\omega} \in \mathbb{C}$ and $\lambda^{1}, \ldots, \lambda^{\omega} \in \mathbb{R}^{s}$ such that

$$
P(x)=\sum_{j=1}^{\omega} c_{j} \mathrm{e}^{i \lambda^{j} \cdot x} \quad\left(x \in \mathbb{R}^{s}\right)
$$

where $\lambda^{1}, \ldots, \lambda^{\omega}$ are distinct and $\omega$ is finite. If every $\dot{c}_{j}(j=1, \ldots, \omega)$ is different from zero, the set

$$
\sigma(P)=\left\{\lambda^{1}, \lambda^{2}, \ldots, \lambda^{\omega}\right\}
$$

is called the spectrum of $P$ and the map

$$
\begin{aligned}
\lambda \rightarrow a(\lambda ; P): & =\lim _{T \rightarrow \infty} \frac{1}{\left|Q_{T}\right|} \int_{Q_{T}} P(x) \mathrm{e}^{-i \lambda \cdot x} d x=: f P(x) \mathrm{e}^{-i \lambda \cdot x} d x \\
& = \begin{cases}c_{j} & \text { if } \lambda=\lambda^{j} \text { for some } j \\
0 & \text { if } \lambda \notin \sigma(P)\end{cases}
\end{aligned}
$$


is called the Bohr transform of $P$, where $Q_{T}=[-T, T]^{s}$ and $\left|Q_{T}\right|=(2 T)^{s}$. For any fixed $q \in[1, \infty)$ we shall denote by $B_{a p}^{q}\left(\mathbb{R}^{s}\right)$ the completion of $\mathcal{P}\left(\mathbb{R}^{s}\right)$ with respect to the norm defined by $\|P\|_{q}=\left(f|P(x)|^{q} d x\right)^{\frac{1}{q}}$ for all $P \in \mathcal{P}\left(\mathbb{R}^{s}\right)$. An element $f \in B_{a p}^{q}\left(\mathbb{R}^{s}\right)$ is defined by a sequence of trigonometric polynomials $\left(P_{n}\right)_{n \in N}$ such that

$$
f=\lim _{n} P_{n} \text { in } B_{a p}^{q}\left(\mathbb{R}^{s}\right) \quad \text { and }\|f\|_{q}:=\left(f|f(x)|^{q} d x\right)^{\frac{1}{q}}=\lim _{n}\left\|P_{n}\right\|_{q} .
$$

Recall that the space $B_{a p}^{\infty}\left(\mathbb{R}^{s}\right):=C_{a p}^{0}\left(\mathbb{R}^{s}\right)$ of all uniformly almost periodic functions is the completion of $\mathcal{P}\left(\mathbb{R}^{s}\right)$ with respect to the $L^{\infty}$-norm

$$
\|P\|_{\infty}=\sup _{x \in \mathbb{R}^{s}}|P(x)| \quad\left(P \in \mathcal{P}\left(\mathbb{R}^{s}\right)\right) .
$$

For these spaces we have the following chain of continuous embeddings, for any $q_{1}, q_{2}>1$ such that $q_{1}<q_{2}<+\infty$ :

$$
C_{a p}^{0}\left(\mathbb{R}^{s}\right)=B_{a p}^{\infty}\left(\mathbb{R}^{s}\right) \hookrightarrow B_{a p}^{q_{2}}\left(\mathbb{R}^{s}\right) \hookrightarrow B_{a p}^{q_{1}}\left(\mathbb{R}^{s}\right) \hookrightarrow B_{a p}^{1}\left(\mathbb{R}^{s}\right)
$$

being $\|f\|_{\infty} \geq\|f\|_{q_{2}} \geq\|f\|_{q_{1}} \geq\|f\|_{1}$ where $\|f\|_{\infty}:=\sup _{x \in \mathbb{R}^{\cdot}}|f(x)|$. For any $f \in$ $B_{a p}^{q}\left(\mathbb{R}^{s}\right)$ we call the map

$$
\lambda \rightarrow a(\lambda ; f):=f f(x) \mathrm{e}^{-i \lambda \cdot x} d x=\lim _{n} a\left(\lambda ; P_{n}\right)
$$

(where the sequence of trigonometric polynomials $\left(P_{n}\right)_{n \in N}$ converges to $f$ in $B_{a p}^{q}\left(\mathbb{R}^{s}\right)$ ) the Bohr transform of $f$.

Definition 2.1. We will call spectrum of the function $f \in B_{a p}^{q}\left(\mathbb{R}^{s}\right)$ the subset of $\mathbb{R}^{s}$ defined by

$$
\sigma(f)=\left\{\lambda \in \mathbb{R}^{s} \mid a(\lambda ; f) \neq 0\right\}
$$

Hence, in particular, when $f$ is the polynomial $P$ given by $(2.1)$, we have $\sigma(P)=$ $\left\{\lambda^{1}, \ldots, \lambda^{\omega}\right\}$.

For the reader's convenience, we report some properties of the spectrum (see [3]). For any $f \in B_{a p}^{q}\left(\mathbb{R}^{s}\right)$ one has:

$$
\begin{aligned}
& \lim _{|\lambda| \rightarrow+\infty} a(\lambda ; f)=0 \\
& \sigma(f) \text { is at most a countable set. } \\
& \sigma(f)=\emptyset \Longleftrightarrow a(\lambda ; f)=0 \text { for all } \lambda \in \mathbb{R}^{s} \Longleftrightarrow f=0 \in B_{a p}^{1}\left(\mathbb{R}^{s}\right) .
\end{aligned}
$$

We call the elements of $\sigma(f)$ Fourier exponents of $f$. Therefore, with each function $f \in B_{a p}^{q}\left(\mathbb{R}^{j}\right)$ we associate formally the Bohr-Fourier series

$$
f \sim \sum_{\lambda \in \sigma(f)} a(\lambda ; f) \mathrm{e}^{i \lambda \cdot x}
$$

As well known, if the series (2.8) is absolutely $\Omega$-convergent with respect to a summation method $\Omega$, then it is unconditionally convergent. 
Remark 2.1. Let $f \in B_{a p}^{1}\left(\mathbb{R}^{s}\right)$. If its Bohr-Fourier series is absolutely convergent, then it is unconditionally uniformly convergent to an uniformly almost periodic function [2: p. 14]. Setting

$$
f^{*}(x)=\sum_{\lambda \in \sigma(f)} a(\lambda ; f) \mathrm{e}^{i \lambda \cdot x} \quad\left(x \in \mathbb{R}^{s}\right)
$$

it results that $a(\lambda ; f)=a\left(\lambda ; f^{*}\right)$ for all $\lambda \in \mathbb{R}^{s}$ and, from (2.7), $\left\|f-f^{*}\right\|_{1}=0$.

Let us recall the Hausdorff-Young theorem for $B_{a p}^{q}$ spaces, which we will use in the proof of the embedding theorems (for the proof, see $[3,4,7]$ ).

Theorem 2.1 (Hausdorff-Young). Let $f \in B_{a p}^{q}\left(\mathbb{R}^{s}\right)$. Then one has

$$
\begin{aligned}
& \left(\sum_{\lambda \in \sigma(f)}|a(\lambda ; f)|^{q^{\prime}}\right)^{\frac{1}{q^{\prime}}} \leq\|f\|_{q} \quad \text { if } q \in(1,2] \\
& \|f\|_{q} \leq\left(\sum_{\lambda \in \sigma(f)}|a(\lambda ; f)|^{q^{\prime}}\right)^{\frac{1}{q^{\prime}}} \quad \text { if } q \in[2,+\infty)
\end{aligned}
$$

where $q^{\prime}=\frac{q}{q-1}$, and the series occurring in (2.10) may be divergent.

\section{Sobolev-Besicovitch spaces $W_{a p}^{m, q}\left(\mathbb{R}^{s}\right)$ and $H_{a p}^{m, q}\left(\mathbb{R}^{s}\right)$}

For any multi-index $\alpha=\left(\alpha_{1}, \ldots, \alpha_{s}\right) \in \mathbb{N}_{0}^{s}$ and $x \in \mathbb{R}^{s}$ we set

$$
\begin{aligned}
|\alpha| & =\alpha_{1}+\ldots+\alpha_{s} & & \\
(x)^{\alpha} & =x_{1}^{\alpha_{1}} \ldots x_{s}^{\alpha_{s}} & & \text { (setting } \left.x_{j}^{\alpha_{j}}=1 \text { if } x_{j}=\alpha_{j}=0\right) \\
\partial^{\alpha} & =\partial_{1}^{\alpha_{1}} \ldots \partial_{s}^{\alpha_{s}} \quad & & \text { (where } \left.\partial_{j} f=\frac{\partial f}{\partial x_{j}}\right) .
\end{aligned}
$$

Let us besides define the sets

$$
J_{m}=\left\{\alpha \in \mathbb{N}_{0}^{s}:|\alpha| \leq m\right\} \quad\left(m \in \mathbb{N}_{0}\right)
$$

The spaces $C_{a p}^{k}\left(\mathbb{R}^{s}\right)$ are naturally defined as the spaces whose elements $f$ are such that $\partial^{\alpha} f \in C_{a p}^{0}\left(\mathbb{R}^{s}\right)$ for all $\alpha \in J_{k}$ equipped with the usual norm, and $C_{a p}^{\infty}\left(\mathbb{R}^{s}\right)=$ $\bigcap_{k=0}^{\infty} C_{a p}^{k}\left(\mathbb{R}^{s}\right)$. For any $q \in[1,+\infty]$ and $m \in \mathbb{N}_{0}$ we set

$$
\begin{aligned}
\|P\|_{W m, \infty} & =\sum_{|\alpha| \leq m}\left\|\partial^{\alpha} P\right\|_{\infty} \\
\|P\|_{W^{m, q}} & =\left(\sum_{|\alpha| \leq m}\left\|\partial^{\alpha} P\right\|_{q}^{q}\right)^{\frac{1}{q}}(q \in[1,+\infty)) \quad\left(P \in \mathcal{P}\left(\mathbb{R}^{s}\right)\right) .
\end{aligned}
$$

Let us observe that, fixed $q,(3.1)$ defines a norm on $\mathcal{P}\left(\mathbb{R}^{s}\right)$ and we have $\|P\| W^{0, q}=\|P\|_{q}$. 
Definition 3.1. For any fixed $q \in[1,+\infty]$ we shall denote by $W_{a p}^{m, q}\left(\mathbb{R}^{s}\right)$ the completion of $\mathcal{P}\left(\mathbb{R}^{s}\right)$ with respect to the norm $\|\cdot\|_{W m .,}$ defined by (3.1). These spaces are called Sobolev-Besicovitch spaces of order $m$ and of type $B^{q}$.

Hence, we can define the norm on the space $W_{a p}^{m, q}$ in the following way:

$$
\|f\|_{W m, q}=\left(\sum_{|\alpha| \leq m}\left\|\partial^{\alpha} f\right\|_{q}^{q}\right)^{\frac{1}{q}} .
$$

Clearly, $W_{a p}^{m, q}\left(\mathbb{R}^{s}\right) \subseteq B_{a p}^{q}\left(\mathbb{R}^{s}\right)$ for all $m \geq 0$ and all $q \geq 1$. According to Definition 3.1, an element $f$ of $W_{a p}^{m, q}\left(\mathbb{R}^{s}\right)$ is defined by means of a sequence $\left(P_{n}\right)_{n \in N}$ of trigonometric polynomials convergent to $f \in B_{a p}^{q}\left(\mathbb{R}^{s}\right)$ and such that, for any multiindex $\alpha$ with $|\alpha| \leq m,\left(\partial^{\alpha} P_{n}\right)_{n \in \mathbb{N}}$ is a Cauchy sequence in $B_{a p}^{q}\left(\mathbb{R}^{s}\right)$. Since the space $B_{a p}^{q}\left(\mathbb{R}^{s}\right)$ is complete, we can set

$$
f_{\alpha}=\lim _{n} \partial^{\alpha} P_{n}
$$

and we will call $f_{\alpha}$ the strong $\alpha$-derivative of $f$, setting $\partial^{\alpha} f=f_{\alpha}$. Observe that for any $\varphi \in C_{a p}^{\infty}\left(\mathbb{R}^{s}\right)$ we get, integrating by parts,

$$
f\left(\partial^{\alpha} P_{n}(x)\right) \varphi(x) d x=(-1)^{|\alpha|} f P_{n}(x) \partial^{\alpha} \varphi(x) d x .
$$

Let us assume $f \in W_{a p}^{m, q}\left(\mathbb{R}^{s}\right)$ and $\alpha \in J_{m}$. The Bohr-Fourier coefficients of $f$ and $f_{\alpha}$ are related by the formula

$$
a\left(\lambda ; f_{\alpha}\right)=i^{|\alpha|}(\lambda)^{\alpha} a(\lambda ; f) \quad(\lambda \in \sigma(f)) .
$$

It follows that $f_{\alpha}$ has the same Bohr-Fourier exponents of $f$, except for $\lambda=0$, if it appears among the Bohr-Fourier exponents of $f$. Therefore, by (2.8) we have

$$
f_{\alpha}(x) \sim \sum_{\lambda \in o(f)} i^{|\alpha|}(\lambda)^{\alpha} a(\lambda ; f) \mathrm{e}^{i \lambda \cdot x} .
$$

Observe that, when $f_{\alpha}$ represents the ordinary derivative of $f$, its Bohr-Fourier series coincides with (3.5).

Definition 3.2. For any fixed $q>1$ and $m \geq 0$ we shall denote by $H_{a p}^{m, q}\left(\mathbb{R}^{s}\right)$ the subspace of $B_{a p}^{1}\left(\mathbb{R}^{s}\right)$ obtained as the completion of $\mathcal{P}\left(\mathbb{R}^{s}\right)$ with respect to the norm defined by

$$
\sum_{\lambda \in \sigma(P)}\left(1+|\lambda|^{2}\right)^{\frac{m e^{\prime}}{2}}|a(\lambda ; P)|^{q^{\prime}}
$$

where $q^{\prime}=\frac{q}{q-1}$. We will call $H_{a p}^{m, q}\left(\mathbb{R}^{s}\right)$ Sobolev-Besicovitch spaces of type $H$.

For any $f \in H_{a p}^{m, q}\left(\mathbb{R}^{s}\right)$ let us introduce the norm

$$
\|f\|_{H^{m, q}}=\left(\sum_{\lambda \in \sigma(f)}\left(1+|\lambda|^{2}\right)^{\frac{m q^{\prime}}{2}}|a(\lambda ; f)|^{q^{\prime}}\right)^{\frac{1}{f^{\prime}}} .
$$


Clearly, for any $m \geq 0$ and $q \geq 2$ we have $H_{a p}^{m, q}\left(\mathbb{R}^{s}\right) \hookrightarrow B_{a p}^{q}\left(\mathbb{R}^{s}\right)$ with continuous embedding. For any $\alpha$ such that $|\alpha| \leq m$ it is possible to show, using the HausdorffYoung theorem, that if $f \in H_{a p}^{m, q}$, then the sequence of polynomials

$$
\partial^{\alpha} Q_{n}=\sum_{\lambda \in \sigma\left(P_{n}\right)} a(\lambda ; f) i^{|\alpha|}(\lambda)^{\alpha} e^{i \lambda \cdot x}
$$

(where $\left(P_{n}\right)_{n \in N}$ is a sequence of polynomials converging to $f$ in $B_{a p}^{q}$ ) converges in $B_{a p}^{q} \quad(q \geq 2)$, and therefore we are able to define, for any $f \in H_{a p}^{m, q}$, the strong derivatives $\partial^{\alpha} f$ as

$$
\partial^{\alpha} f=\lim _{n} \partial^{\alpha} Q_{n} \quad \text { in } B_{a p}^{q} .
$$

It is easy to show the following properties for the $H_{a p}^{m, p}$ spaces, which will be used in what follows.

Proposition 3.1. Let $q \geq 2$ and $|\alpha|=k \leq m$. If $f \in H_{a p}^{m, q}\left(\mathbb{R}^{s}\right)$, then $\partial^{\alpha} f \in$ $H_{a p}^{m-k, q}\left(\mathbb{R}^{s}\right)$.

Proposition 3.2. Let $q \geq 2$ and $|\alpha|=k$. If $f \in H_{a p}^{m, q}\left(\mathbb{R}^{s}\right)$ and $\partial^{\alpha} f \in H_{a p}^{m, q}\left(\mathbb{R}^{s}\right)$, then $f \in H_{a p}^{m+k, q}\left(\mathbb{R}^{s}\right)$.

\section{Embeddings between $H_{a p}^{m, q}$ and $W_{a p}^{m, q}$ spaces}

Let us prove the following

Theorem 4.1. Let $m \in \mathbb{N}$ be arbitrary. Then:

(i) For all $q \in(1,2]$, we have $W_{a p}^{m, q}\left(\mathbb{R}^{s}\right) \hookrightarrow H_{a p}^{m, q}\left(\mathbb{R}^{s}\right)$.

(ii) For all $q \geq 2$, we have $H_{a p}^{m, q}\left(\mathbb{R}^{s}\right) \hookrightarrow W_{a p}^{m, q}\left(\mathbb{R}^{s}\right)$.

Proof. (i) Let $f \in W_{a p}^{m, q}\left(\mathbb{R}^{9}\right)$. If $m=0$, the thesis follows from the HausdorffYoung theorem. Now, let $m \geq 1$. Since $\frac{m}{2} q^{\prime} \geq 1$, we have

$$
\|f\|_{H^{m, q}}^{q^{\prime}} \leq k\left(\sum_{\lambda \in \sigma(f)}\left(1+|\lambda|^{m q^{\prime}}\right)|a(\lambda ; f)|^{q^{\prime}}\right) \leq k \sum_{\nu=0}^{m}\left(\sum_{\lambda \in \sigma(f)}|\lambda|^{\nu q^{\prime}}|a(\lambda ; f)|^{q^{\prime}}\right)
$$

where $k=2^{\frac{m}{2} q^{\prime}-1}$. On the other hand, since $\left\|\partial^{\alpha} f\right\|_{q}<+\infty$, by the Hausdorff-Young theorem we have, for all $\nu=1, \ldots, m$,

$$
\left(\sum_{|\alpha|=\nu}\left\|\partial^{\alpha} f\right\|_{q}\right)^{q^{\prime}} \geq \sum_{|\alpha|=\nu}\left\|\partial^{\alpha} f\right\|_{q}^{q^{\prime}} \geq \sum_{\lambda \in \sigma(f)}|a(\lambda ; f)|^{q^{\prime}}\left(\sum_{|\alpha|=\nu}\left|(\lambda)^{\alpha}\right|^{q^{\prime}}\right) .
$$

Besides, we have, for a suitable $C \in \mathbb{R}_{+}{ }^{1}$ )

$$
\| f{\| W m_{:} \bullet} \geq C \sum_{\nu=0}^{m}\left(\sum_{\lambda \in \sigma(f)}|a(\lambda, f)|^{q^{\prime}}|\lambda|^{q^{\prime} \nu}\right)^{\frac{1}{q}} .
$$

1) Estimate (4.1) follows from the fact that there exist $\chi_{0}, \chi_{1} \in \mathbb{R}_{+}$such that, for all $\lambda \in$ $\mathbb{R}^{0}$ and $\nu \in \mathbb{N}_{0}, \chi_{0}|\lambda|^{2 \nu} \leq \sum_{|\alpha|=\nu}\left|(\lambda)^{\alpha}\right|^{2} \leq \chi_{1}|\lambda|^{2 \nu}$. Then, in this case, we have $C=$ $\chi_{0}^{\frac{1}{2}} 2^{\left(\frac{1}{2}-\frac{1}{q}\right)\left(1-p_{m}\right)}$, where $p_{m}=p(s, m)$ is the number of vectors $\alpha$ such that $|\alpha|=m$. 
Therefore, there exists $K \in \mathbb{R}_{+}$such that $\|f\|_{H^{m, q}} \leq K\|f\|_{W^{m, q}}$.

(ii) From the Hausdorff-Young theorem it follows that

$$
\|f\|_{q} \leq\left(\sum_{\lambda \in \sigma(f)}|a(\lambda ; f)|^{q^{\prime}}\right)^{\frac{1}{\gamma^{\prime}}}
$$

hence, since, by Proposition 3.1, $\partial^{\alpha} f \in B_{a p}^{q}$ for all $\alpha \in J_{m}$,

$$
\|f\| W_{m, q} \leq \sum_{\alpha \in J_{m}}\left(\sum_{\lambda \in \sigma(f)}\left|(\lambda)^{\alpha}\right|^{q^{\prime}}|a(\lambda ; f)|^{q^{\prime}}\right)^{\frac{1}{q^{\prime}}}
$$

Since $\left|(\lambda)^{\alpha}\right|^{q^{\prime}} \leq|\lambda|^{m q^{\prime}} \leq\left(1+|\lambda|^{2}\right)^{\frac{m}{2} q^{\prime}}$, we have

$$
\|f\|_{W_{m, q}} \leq \sum_{\alpha \in J_{m}}\left(\sum_{\lambda \in \sigma(f)}\left(1+|\lambda|^{2}\right)^{\frac{m}{2} q^{\prime}}|a(\lambda ; f)|^{q^{\prime}}\right)^{\frac{1}{q^{\prime}}}=\bar{p}\|f\|_{H^{m, q}}
$$

where $\bar{p}=\sum_{\nu=1}^{m} p(s, \nu) \mathbf{\square}$

From Theorem 4.1 we obtain the relation $H_{a p}^{m, 2}=W_{a p}^{m, 2} \quad(m \in \mathbb{N})$ which is also a trivial consequence of the Parseval equality.

\section{Embedding theorems for $H_{a p}^{m, q}$ spaces}

We are going to establish sufficient conditions in order to guarantee some embedding results. Let us fix a set $\Lambda \subset \mathbb{R}^{3} \backslash\{0\}$ satisfying the following properties:

a) $\operatorname{card} \Lambda=\operatorname{card} \mathbb{N}$.

b) $\Lambda$ is ordered with respect to the absolute value: $\Lambda=\left\{\lambda^{n}: n \in \mathbb{N}\right\}$ with $\left|\lambda^{i}\right| \leq\left|\lambda^{j}\right|$ for $i \leq j$.

c) $\Lambda$ has a unique limit point and this is the point at infinity.

d) There exists $\beta \geq 0$ such that

$$
\sum_{\lambda \in \Lambda} \frac{1}{|\lambda|^{\gamma}}<+\infty \quad(\gamma>\beta) .
$$

We shall consider almost periodic functions such that $\sigma(f) \subseteq \Lambda$. Let us note that $\Lambda \subset \mathbb{R}^{s} \backslash\{0\}$ means that, for the sake of simplicity and without loss of generality, we consider almost periodic functions with asymptotic mean equal to zero. Observe that these classes of almost periodic functions include the periodic functions and the quasiperiodic ones that are obtained as finite sums of periodic functions, whose generators are rationally independent. In fact, in this case we have $\beta=s$, since the series (5.1) has the same behaviour of the multiple generalized harmonic series $\sum_{k \in Z} \frac{1}{|k|^{\gamma}}$ where $\mathbb{Z}_{*}^{s}=\mathbb{Z}^{s} \backslash\{(0, \ldots, 0)\}$. 
Let us denote by $C_{a p}^{0, \mu}\left(\mathbb{R}^{3}\right)$ the space of those uniformly almost periodic functions that are Hölderian too, i.e.

$$
C_{a p}^{0, \mu}\left(\mathbb{R}^{s}\right)=\left\{f \in C_{a p}^{0}\left(\mathbb{R}^{s}\right):[f]_{\mu}:=\sup _{x \neq y} \frac{|f(x)-f(y)|}{|x-y|^{\mu}}<+\infty\right\}
$$

equipped with the usual norm. Finally, let us set

$$
B_{a p}^{q}(\Lambda)=\left\{f \in B_{a p}^{q}\left(\mathbb{R}^{s}\right): \sigma(f) \subseteq \Lambda\right\}
$$

and, analogously, $W_{a p}^{m, q}(\Lambda), H_{a p}^{m, q}(\Lambda)$ and $C_{a p}^{0, \mu}(\Lambda)$. Observe that these spaces are separable.

We have the following embedding results.

Theorem 5.1. Suppose that $\Lambda \subset \mathbb{R}^{s} \backslash\{0\}$, with $\operatorname{card} \Lambda=\operatorname{card} \mathbb{N}$, satisfies (5.1). Then:

(i) If $m q<\beta$ and either a) $q \geq 2$ or b) $1<q<2$ and $\beta<\frac{2 m q}{2-q}$, we have

$$
H_{a p}^{m, q}(\Lambda) \hookrightarrow B_{a p}^{r}(\Lambda) \quad \text { for all } r \in\left[q, \frac{\beta q}{\beta-m q}\right) \text {. }
$$

(ii) If $\dot{m q}=\beta$, we have $H_{a p}^{m, q}(\Lambda) \hookrightarrow B_{a p}^{r}(\Lambda)$ for all $r \in[q,+\infty)$.

(iii) If $m q>\beta$, we have $H_{a p}^{m, q}(\Lambda) \hookrightarrow C_{a p}^{0}(\Lambda)$.

(iv) If $m q>\beta \geq(m-1) q$, we have $H_{a p}^{m, q}(\Lambda) \hookrightarrow C_{a p}^{0, \mu}(\Lambda)$ for all $\mu \in\left(0, m-\frac{\beta}{q}\right)$.

Proof. (i) Let us consider, first of all, the case $q \geq 2$. By the Hölder inequality, we get

$$
\begin{aligned}
& \sum_{j=n+1}^{n+p}\left|a\left(\lambda^{j} ; f\right)\right|^{r^{\prime}} \\
& \quad \leq\left(\sum_{j=n+1}^{n+p}\left(\left|a\left(\lambda^{j} ; f\right)\right|\left|\lambda^{j}\right|^{m}\right)^{q^{\prime}}\right)^{\frac{r^{\prime}}{q^{\prime}}}\left(\sum_{j=n+1}^{n+p} \frac{1}{\left|\lambda^{j}\right|^{\frac{m g r}{r-q}}}\right)^{\frac{r-q}{\sigma(r-1)}} \\
& \leq\left(\sum_{j=n+1}^{n+p}\left|a\left(\lambda^{j} ; f\right)\right|^{q^{\prime}}\left(1+\left|\lambda^{j}\right|^{2}\right)^{\frac{m}{2} q^{\prime}}\right)^{\frac{r^{\prime}}{\sigma^{\prime}}}\left(\sum_{j=n+1}^{n+p} \frac{1}{\left|\lambda^{j}\right|^{\frac{m g r}{r-q}}}\right)^{\frac{r-g}{q(r-1)}}
\end{aligned}
$$

for all $p \in \mathbb{N}$. From convergence of (3.6) and of (5.1), we can say that the first term in (5.2) is a Cauchy series and, consequently, is convergent, provided $\frac{m q r}{r-q}>\beta$, that is

$$
r<\frac{\beta q}{\beta-m q} .
$$

In this case, being $r \geq 2$, from the Hausdorff-Young theorem it follows that $\|f\|_{r} \leq$ $C\|f\|_{H^{m . q}}$ where $C=\left[\sum_{j=1}^{\infty}\left|\lambda^{j}\right|^{-\frac{m r q}{r-q}}\right]^{\frac{r-q}{r q}}$, i.e. the thesis. 
Let now $q<2$. We may consider $r \geq 2$. Hence, we must have $\frac{\beta q}{\beta-m q}>2$, that is

$$
\beta<\frac{2 m q}{2-q}
$$

Condition (5.4) is then sufficient to guarantee the proof when $q \in(1,2)$. Indeed, we have

$$
H_{a p}^{m, q} \hookrightarrow B_{a p}^{r} \hookrightarrow B_{a p}^{q}
$$

with $1<q<2 \leq r$.

(ii) When $q \geq 2$, we have

$$
\sum_{j=n+1}^{n+p}\left|a\left(\lambda^{j} ; f\right)\right|^{r^{\prime}} \leq\left(\sum_{j=n+1}^{n+p}\left|a\left(\lambda^{j} ; f\right)\right|^{q^{\prime}}\left|\lambda^{j}\right|^{q^{\prime} m}\right)^{\frac{r^{\prime}}{q^{\prime}}}-\left(\sum_{j=n+1}^{n+p} \frac{1}{\left|\lambda^{j}\right|^{m r^{\prime}\left(\frac{g^{\prime}}{r^{\prime}}\right)^{\prime}}}\right)^{\frac{1}{\left(\frac{g^{j}}{r^{\prime}}\right)^{\prime}}} .
$$

The thesis follows whenever $\frac{\beta r}{r-q}>\beta$, that is $r>q$. Let us, again, consider $1<q<2$ and $r \geq 2$. The thesis follows immediately from (5.5).

(iii) We have

$$
\sum_{j=n+1}^{n+p}\left|a\left(\lambda^{j} ; f\right)\right| \leq\left(\sum_{j=n+1}^{n+p}\left|a\left(\lambda^{j} ; f\right)\right|^{q^{\prime}}\left|\lambda^{j}\right|^{m q^{\prime}}\right)^{\frac{1}{q}}\left(\sum_{j=n+1}^{n+p} \frac{1}{\left|\lambda^{j}\right|^{m q}}\right)^{\frac{1}{q}} .
$$

From (3.6) and the hypothesis $m q>\beta$, it follows that

$$
\sum_{j=1}^{\infty}\left|a\left(\lambda^{j} ; f\right)\right| \leq L\|f\|_{H^{m, q}} \quad \text { where } L=\left(\sum_{j=1}^{\infty} \frac{1}{\left|\lambda^{j}\right|^{m q}}\right)^{\frac{1}{q}}
$$

Note that $f \in B_{a p}^{q}\left(\mathbb{R}^{s}\right)$ and hence $f \in B_{a p}^{l}\left(\mathbb{R}^{s}\right)$. Being the Bohr-Fourier series of $f$ absolutely convergent, then it converges unconditionally uniformly to a function $f^{*} \in$ $C_{a p}^{0}\left(\mathbb{R}^{s}\right)$, such that $\left\|f^{*}\right\|_{\infty} \leq \sum_{j=1}^{\infty}\left|a\left(\lambda^{j} ; f\right)\right|$ and $\left\|f^{*}-f\right\|_{1}=0$. Therefore, we claim that $f \in C_{a p}^{0}\left(\mathbb{R}^{s}\right)$.

(iv) We have (see, for example, [3])

$$
[f]_{\mu} \leq \sum_{j=1}^{\infty}\left|a\left(\lambda^{j} ; f\right)\right| 2^{1-\mu}\left|\lambda^{j}\right|^{\mu}=2^{1-\mu} \sum_{j=1}^{\infty}\left|a\left(\lambda^{j} ; f\right)\right|\left|\lambda^{j}\right|^{\mu} .
$$

By the Hölder inequality, we have

$$
\sum_{j=n+1}^{n+p}\left|a\left(\lambda^{j} ; f\right)\right|\left|\lambda^{j}\right|^{\mu} \leq\left(\sum_{j=n+1}^{n+p}\left|a\left(\lambda^{j} ; f\right)\right|^{q^{\prime}}\left|\lambda^{j}\right|^{m q^{\prime}}\right)^{\frac{1}{q}}\left(\sum_{j=n+1}^{n+p} \frac{1}{\left|\lambda^{j}\right|^{(m-\mu) q}}\right)^{\frac{1}{q}} .
$$

By (3.6) and (5.1), the thesis will follow whenever $\mu<m-\frac{\beta}{q}$ 
Remark 5.1. Observe that, proving statement (iii) of Theorem 5.1, we have proved something more, i.e. that, if $m q>\beta$, then $\sum_{j=1}^{\infty}\left|a\left(\lambda^{j} ; f\right)\right|<+\infty$. This is a generalization of a result given by Stein and Weiss [13: p. 249] in the context of periodic functions of the class $C^{m}$ in the case $q=2$.

Remark 5.2. The proof of Theorem 5.1 shows the fundamental importance of hypothesis (5.1) to obtain better embeddings than those ones that we would have independently from the structure of the spectrum.

From Proposition 3.1 and Theorem 5.1 we easily get

Corollary 5.1. If $m q>\beta$ and $q \geq 2$, then $H_{a p}^{m+n, q}(\Lambda) \hookrightarrow C_{a p}^{n}(\Lambda)$ for all $n \in \mathbb{N}$.

Remark 5.3. In condition (5.1) the unboundedness of $\Lambda$, as well as the existence of a unique accumulation point at infinity, plays a decisive role for the embeddings stated in Theorem 5.1. In fact, it is easy to prove that, for all $r \geq 0$ and $q>1, H_{a p}^{r, q}(\Lambda)=$ $H_{a p}^{\infty, q}(\Lambda):=\bigcap_{m \geq 0} H_{a p}^{m, q}(\Lambda)$ whenever $\Lambda$ is a bounded subset of $\mathbb{R}^{s}$. Nevertheless, in this case $H_{a p}^{\infty, q}(\Lambda)$ is not embedded even in $C_{a p}^{0}$. Indeed, there are elements of $H_{a p}^{\infty, q}(\Lambda)$ that are not continuous. Consider, for example, the function having the Bohr-Fourier series

$$
f \sim \sum_{k=1}^{\infty} \frac{1}{k^{\eta}} \mathrm{e}^{i \lambda^{k} \cdot x}
$$

with $\eta \in\left(\frac{1}{2}, 1\right], \lambda^{k} \mathbb{Z}$-linearly independent and such that $\left|\lambda^{k}\right| \leq M$ for all $k \in \mathbb{N}$. We have

$$
\|f\|_{H_{a, p}^{0,2}}^{2}=\sum_{k=1}^{\infty} \frac{1}{k^{2 \eta}}<+\infty .
$$

Hence $f \in H_{a p}^{0,2}(\Lambda)$ and, therefore, $f \in H_{a p}^{\infty, 2}(\Lambda)$. Now if we suppose $f \in C_{a p}^{0}(\Lambda)$, we should have $\sum_{k=1}^{\infty}\left|a\left(\lambda^{k} ; f\right)\right|<+\infty$ (cp. [10: p. 19]), while the series $\sum_{k=1}^{\infty} \frac{1}{k^{\eta}}$ is divergent, being $\eta \in\left(\frac{1}{2}, 1\right]$.

From Theorems 4.1 and 5.1 we have immediately

Corollary 5.2. Suppose that $\Lambda \subseteq \mathbb{R}^{s} \backslash\{0\}$, with card $\Lambda=$ card $\mathbb{N}$, satisfies property (5.1), and let $q \in(1,2]$. Then:

(i) If $m q<\beta<\frac{2 m q}{2-q}$, we have $W_{a p}^{m, q}(\Lambda) \hookrightarrow B_{a p}^{r}(\Lambda)$ for all $r \in\left[q, \frac{\beta q}{\beta-m q}\right)$.

(ii) If $m q=\beta$, we have $W_{a p}^{m, q}(\Lambda) \hookrightarrow B_{a p}^{r}(\Lambda)$ for all $r \geq q$.

(iii) If $m q>\beta$, we have $W_{a p}^{m, q}(\Lambda) \hookrightarrow C_{a p}^{0}(\Lambda)$.

(iv) If $m q>\beta \geq(m-1) q$, we have $W_{a p}^{m, q}(\Lambda) \hookrightarrow C_{a p}^{0, \mu}(\Lambda)$ for all $\mu \in\left(0, m-\frac{\beta}{q}\right)$.

Remark 5.4. Theorem 5.1 and Corollary $5.2 /$ (iv) give embedding also in the case $\Lambda \neq \mathbb{Z}_{*}^{\text {; }}$ for example, if $\Lambda=\left(n\left(1+\frac{1}{|n|^{2}}\right)\right)_{n \in Z_{:}}$, then $\beta$ is equal to $s$ and Theorem $5.1 /(\mathrm{iv})$ holds true, claiming moreover that the Bohr-Fourier series is unconditionally uniformly convergent. Observe that in the periodic case the embedding holds also with $\mu=m-\frac{\beta}{q}$. For almost periodic functions this is an open problem. 


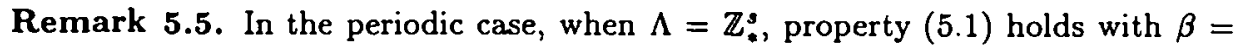
$s$ and Corollary 5.2 becomes one of the famous Sobolev embedding theorems in the particular case $q \in[1,2]$ (see [1: Theorem 5.4]). When $q>2$, we have, at the moment, the embeddings that follow from $W_{a p}^{m, q} \hookrightarrow W_{a p}^{m, 2}$. We expect, anyway, to find better embeddings, being for $q>2$ the spaces $W_{a p}^{m, q}$ more regular than $W_{a p}^{m, 2}$. It is our opinion that these embeddings can be improved via "contiguity" properties between the spaces of type $W$ and $H$.

Remark 5.6. Given $B_{a p}^{q}$ and fixed $m$, let us consider two spectra $\Lambda_{1}$ and $\Lambda_{2}$. We can say that if $\Lambda_{1}$ is thicker than $\Lambda_{2}$, it is necessary an exponent $\beta_{1}$ greater than $\beta_{2}$ to obtain the convergence of the series (5.1). But, since $\frac{\beta_{1 q}}{\beta_{1}-m q}<\frac{\beta_{2} q}{\beta_{2}-m q}$ if $\beta_{1}>\beta_{2}$, the thicker is $\Lambda$, the less regular is the embedding of the space $H_{a p}^{m, q}(\Lambda)$.

Let us recall now that a trigonometric series is said to be lacunary if it has the form

$$
\sum_{k=1}^{\infty} c_{k} \mathrm{e}^{i n_{k} \cdot x}
$$

where the natural numbers $n_{k}$ satisfy the inequality $\frac{n_{k+1}}{n_{k}} \geq p>1$ for all $k \in \mathbb{N}$. We want to extend this notion to Bohr-Fourier series, when $s=1$.

Definition 5.1. Given a series $\sum_{j=1}^{\infty} c_{\lambda j} e^{i \lambda^{j} x}$ with $\lambda^{j},\left|\lambda^{1}\right| \leq\left|\lambda^{2}\right| \leq \ldots$, and $x \in \mathbb{R}$, we will say that it is lacunary if there exists $p>1$ such that $\frac{\left|\lambda^{j+1}\right|}{\left|\lambda^{j}\right|} \geq p$ for all $j \in \mathbb{N}$.

Let us prove the following

Proposition 5.1. If $f \in H_{a p}^{m, q}(\Lambda)$ with $\Lambda \subseteq \mathbb{R} \backslash\{0\}$ for some $m>0, q>1$ and its Bohr-Fourier series is lacunary, then $f \in C_{a p}^{0}(\Lambda)$. Moreover, if $q \geq 2$, then $f \in C_{a p}^{r}(\Lambda)$ for any $r<m$.

Proof. For every lacunary series, property (5.1) holds for any $\gamma>0$; hence $\beta=0$ and we get $f \in C_{a p}^{0}(\Lambda)$. By Proposition 3.1, if $f \in H^{m, q}(\Lambda)$ with $q \geq 2$, then $\partial^{\alpha} f \in$ $H^{m-|\alpha|, q}(\Lambda)$ for any $\alpha$ such that $|\alpha| \leq m$. Besides, we have $\sigma\left(\partial^{\alpha} f\right) \subseteq \sigma(f)$ and so the Bohr-Fourier series of $\partial^{\alpha} f$ is lacunary, too. It follows that for any $\alpha$ such that $|\alpha|<m$ we have $\partial^{\alpha} f \in C_{a p}^{0}(\Lambda)$, i.e. the thesis

\section{6. $\beta$ as the "dimension" of an additive semigroup}

In the previous section we remarked that, in the periodic case and for a particular class of quasi-periodic functions, $\beta$ is equal to the dimension $s$ of the space where they are defined. This is not true for general almost periodic functions, even for the simplest quasi-periodic case. Here we shall consider the class of quasi-periodic functions whose spectrum $\Lambda^{\star}$ is an additive semigroup, generated by a finite number of $\mathbb{Z}$-linearly independent frequencies belonging to a convex semicone of $\mathbb{R}^{s}$. For this class, we shall prove that $\beta$ is equal to the number of generators of the semigroup; moreover that, whenever $2 m>\beta$, the spaces $W_{a p}^{m, 2}\left(\Lambda^{*}\right)$ become Banach algebras, generalizing a classical result on Sobolev spaces (see [1: p. 115]). 
Lemma 6.1. Let

$$
\Lambda^{\star}=\left\{\left\{n_{1} \lambda_{\star}^{1}+\ldots+n_{\beta} \lambda_{\star}^{\beta}\right\}_{n_{j} \in N_{0}}: n_{1}+\ldots+n_{\beta}>0\right\}
$$

where $\lambda_{\star}^{1}, \ldots, \lambda_{\star}^{\beta} \in \mathbb{R}^{s}$ are $\mathbb{Z}$-linearly independent and such that

$$
\overline{\operatorname{conv}}\left\{\lambda_{\star}^{1}, \ldots, \lambda_{\star}^{\beta}\right\} \cap\{0\}=\emptyset \text {. }
$$

Then one has

$$
\sum_{\lambda \in \Lambda^{*}} \frac{1}{|\lambda|^{\gamma}} \begin{cases}<+\infty & \text { if } \gamma>\beta \\ =+\infty & \text { if } \gamma \leq \beta\end{cases}
$$

Proof. Let us consider the set

$$
\Gamma^{*}=\left\{\left\{r_{1} \lambda_{*}^{1}+\ldots+r_{\beta} \lambda_{*}^{\beta}\right\}_{r_{i} \in \mathbb{R}_{+}}: r_{1}^{2}+\ldots+r_{\beta}^{2}=1\right\} .
$$

Then $\Gamma^{*} \subset \overline{\operatorname{conv}}\left\{\lambda_{\star}^{1}, \ldots, \lambda_{\star}^{\beta}\right\}$. If we set $K_{\star}=\inf \left\{|y|: y \in \Gamma^{\star}\right\}$, condition (6.1) implies that $K_{*}>0$. Setting $|n|=\sqrt{n_{1}^{2}+\ldots+n_{\beta}^{2}}$ we have, for any $\lambda \in \Lambda^{*}$,

$$
|\lambda|=\left|n_{1} \lambda_{\star}^{1}+\ldots+n_{\beta} \lambda_{\star}^{\beta}\right|=|n|\left|\frac{n_{1}}{|n|} \lambda_{\star}^{1}+\ldots+\frac{n_{\beta}}{|n|} \lambda_{\star}^{\beta}\right| \geq K_{\star}|n| .
$$

On the other hand,

$$
|\lambda| \leq n_{1}\left|\lambda_{\star}^{1}\right|+\ldots+n_{\beta}\left|\lambda_{\star}^{\beta}\right| \leq\left[\sum_{j=1}^{\beta}\left(n_{j}\right)^{2}\right]^{\frac{1}{2}}\left[\sum_{j=1}^{\beta}\left|\lambda_{\star}^{j}\right|^{2}\right]^{\frac{1}{2}}=C_{\star}|n| .
$$

Therefore

$$
\left(\frac{1}{C_{\star}}\right)^{\gamma} \frac{1}{|n|^{\gamma}} \leq \frac{1}{|\lambda|^{\gamma}} \leq\left(\frac{1}{K_{\star}}\right)^{\bar{\gamma}} \frac{1}{|n|^{\gamma}}
$$

i.e. the thesis

Remark 6.1. Let us observe that, being $\Lambda^{*}$ an additive semigroup, it is easy to see that, if $P, Q \in \mathcal{P}\left(\Lambda^{*}\right)$, then $P Q \in \mathcal{P}\left(\Lambda^{*}\right)$.

Proposition 6.1. If $2 m>\beta$, then there exists a constant $K^{*}$ depending on $m$ and $\beta$ such that, for any $P, Q \in \mathcal{P}\left(\Lambda^{*}\right)$, the product $P Q$ satisfies

$$
\|P Q\|_{W^{m, 2}} \leq K^{*}\|P\|_{W^{m, 2}}\|Q\|_{W^{m, 2}} .
$$

Proof. It is sufficient to show that, for any multi-index $\alpha$ with $|\alpha| \leq m$, we have

$$
f\left|\partial^{\alpha}(P(x) Q(x))\right|^{2} d x \leq K_{\alpha}\|P\|_{W^{m, 2}}\|Q\|_{W^{m, 2}}
$$

By the Leibniz rule we obtain

$$
\partial^{\alpha}(P(x) Q(x))=\sum_{\eta \leq \alpha}\left(\begin{array}{l}
\alpha \\
\eta
\end{array}\right) \partial^{\eta} P(x) \partial^{\alpha-\eta} Q(x)
$$


Therefore it is sufficient to show that for any multi-index $\eta \leq \alpha$ with $|\alpha| \leq m$ we have

$$
f\left|\partial^{\eta} P(x) \partial^{\alpha-\eta} Q(x)\right|^{2} d x \leq K_{\eta, \alpha}\|P\|_{W^{m, 2}}\|Q\|_{W^{m, 2}}
$$

To this end, we shall divide the proof in two steps.

Step (i): By the embedding theorems, for any $\eta$ such that $|\eta| \leq m$ there exists a constant $K(\eta)=K(\eta, m, \beta)$ such that for any $R \in \mathcal{P}\left(\Lambda^{\star}\right)$ we get

$$
f\left|\partial^{\eta} R(x)\right|^{r} d x \leq K(\eta)\|R\|_{W m, 2}^{r}
$$

provided $2(m-|\eta|)<\beta$, where $r \in\left[2, \frac{2 \beta}{\beta-2(m-|\eta|)}\right)$. When $2(m-|\eta|)=\beta$, inequality (6.3) holds with $r \in[2,+\infty)$. If it happens that $2(m-|\eta|)>\beta$, we have

$$
\left|\partial^{\eta} R(x)\right| \leq K(\eta)\|R\|_{W m, 2}
$$

a.e. in $\mathbb{R}^{*}$.

Step (ii): Let $l$ be the largest integer such that $2(m-l)>\beta$. Since $2 m>\beta$, we have $l \geq 0$. Now, we have three cases.

A) If $|\eta| \leq l$, then $2(m-|\eta|)>\beta$ and therefore, by (6.4), we have

$$
\begin{aligned}
f\left|\partial^{\eta} P(x) \partial^{\alpha-\eta} Q(x)\right|^{2} d x & \leq[K(\eta)]^{2}\|P\|_{W^{m, 2}}\left\|\partial^{\alpha-\eta} Q\right\|_{2} \\
& \leq[K(\eta)]^{2}\|P\|_{W^{m, 2}}\|Q\|_{W^{m, 2}}
\end{aligned}
$$

B) If $|\alpha-\eta| \leq l$, still by (6.4) we get

$$
f\left|\partial^{\eta} P(x) \partial^{\alpha-\eta} Q(x)\right|^{2} d x \leq[K(\alpha-\eta)]^{2}\|P\|_{W^{m, 2}}\|Q\|_{W^{m, 2}}
$$

C) If $|\eta|>l$ and $|\alpha-\eta|>l$, then $|\eta| \geq l+1$ and $|\alpha-\eta| \geq l+1$; hence $\beta \geq 2(m-|\eta|)$ and $\beta \geq 2(m-|\alpha-\eta|)$. Moreover,

$$
\frac{\beta-2(m-|\eta|)}{\beta}+\frac{\beta-2(m-|\alpha-\eta|)}{\beta}=2-\frac{2(2 m-|\alpha|)}{\beta} \leq 2-\frac{2 m}{\beta}<1 .
$$

Hence there exist $r, r^{\prime}>1$ with $\frac{1}{r}+\frac{1}{r^{\prime}}=1$ such that

$$
1<r<\frac{\beta}{\beta-2(m-|\eta|)} \quad \text { and } \quad 1<r^{\prime}<\frac{\beta}{\beta-2(m-|\alpha-\eta|)}
$$

Thus by the Hölder inequality and (6.3) we get

$$
\begin{aligned}
f\left|\partial^{\eta} P(x) \partial^{\alpha-\eta} Q(x)\right|^{2} d x & \leq\left(f\left|\partial^{\eta} P(x)\right|^{2 r} d x\right)^{\frac{1}{r}}\left(f\left|\partial^{\alpha-\eta} Q(x)\right|^{2 r^{\prime}} d x\right)^{\frac{1}{r}} \\
& \leq[K(\eta)]^{\frac{1}{r}}[K(\alpha-\eta)]^{\frac{1}{r}}\|P\|_{W^{m, 2}}^{2}\|Q\|_{W^{m, 2}}^{2} .
\end{aligned}
$$

The thesis follows immediately 
Theorem 6.1. If $2 m>\beta$, then there exists a constant $K^{*}$ depending on $m$ and $\beta$ such that for any $u, v \in W_{a p}^{m, 2}\left(\Lambda^{*}\right)$ we have that $u v \in W_{a p}^{m, 2}\left(\Lambda^{*}\right)$ and

$$
\|u v\|_{m, 2} \leq K^{*}\|u\|_{W^{m, 2}}\|v\|_{W^{m, 2}}
$$

In particular, $W_{a p}^{m, 2}\left(\Lambda^{*}\right)$ is a commutative Banach algebra with respect to pointwise multiplication and the equivalent norm $\|\cdot\|_{W^{m, 2}}^{*}=K^{*}\|\cdot\| W_{m, 2}$.

Proof. Being $u, v \in W_{a p}^{m, 2}\left(\Lambda^{*}\right)$, there exist sequences $\left(u_{n}\right)_{n \in \mathbb{N}}$ and $\left(v_{n}\right)_{n \in \mathbb{N}}$ in $\mathcal{P}\left(\Lambda^{*}\right)$ converging to $u$ and $v$ in $W_{a p}^{m, 2}$, respectively. By Proposition 6.1 and Remark 6.1 we have that $\left(u_{n} v_{n}\right)_{n \in N}$ is a Cauchy sequence in $W_{a p}^{m, 2}\left(\Lambda^{*}\right)$ and therefore it converges to an element of the space. Being $2 m>\beta$, by the embedding theorems $u$ and $v$ may be assumed continuous and we have

$$
\begin{aligned}
\left\|u_{n} v_{n}-u v\right\|_{2} & \leq\left\|u_{n}\left(v_{n}-v\right)\right\|_{2}+\left\|v\left(u_{n}-u\right)\right\|_{2} \\
& \leq\left\|u_{n}\right\|_{0}\left\|v_{n}-v\right\|_{2}+\|v\|_{0}\left\|u_{n}-u\right\|_{2} \\
& \rightarrow 0
\end{aligned}
$$

when $n \rightarrow \infty$, and therefore $u_{n} v_{n} \rightarrow u v$ in $B_{a p}^{2}$. Defining, for any multi-index $\alpha$ with $|\alpha| \leq m$,

$$
\partial^{\alpha}(u v)=\lim _{n \rightarrow \infty} \partial^{\alpha}\left(u_{n} v_{n}\right)
$$

in $B_{a p}^{2}$, we have that this limit exists, being $\left(u_{n} v_{n}\right)$ convergent in $W_{a p}^{m, 2}$. Finally, we have

$$
\begin{aligned}
\|u v\|_{W^{m, 2}} & =\lim _{n \rightarrow \infty}\left\|u_{n} v_{n}\right\|_{W^{m, 2}} \\
& \leq \lim _{n \rightarrow \infty} K^{*}\left\|u_{n}\right\| W_{W^{m, 2}}\left\|v_{n}\right\|_{W^{m, 2}} \\
& =K^{*}\|u\|_{W^{m, 2}}\|v\|_{W^{m, 2}}
\end{aligned}
$$

In particular,

$$
\begin{aligned}
\|u v\|_{W^{m, 2}}^{*} & =K^{*}\|u v\|_{W^{m, 2}} \\
& \leq K^{*}\left(K^{*}\|u\|_{W^{m, 2}}\|v\|_{W^{m, 2}}\right) \\
& =\|u\|_{W^{m, 2}}^{*}\|v\|_{W^{m, 2}}^{*}
\end{aligned}
$$

i.e. the thesis

Acknowledgements. The authors are deeply grateful to Prof. A. Avantaggiati for his precious suggestions and encouragements. 


\section{References}

[1] Adams, R. A.: Sobolev Spaces. New York et al.: Acad. Press 1975.

[2] Amerio, L. and G. Prouse: Almost Periodic Functions and Functional Equations. New York et al.: Van Nostrand Reinhold 1971.

[3] Avantaggiati, A., Bruno, G. and R. Iannacci: The Hausdorff-Young theorem for almost periodic functions and some applications. Nonlin. Anal. 25 (1995), $61-87$.

[4] Avantaggiati, A.: Il teorema di Hausdorff-Young negli spazi $B_{a p}^{q}$ di funzioni quasi periodiche secondo Besicovitch con applicazioni. Conf. Sem. Mat. Univ. Bari 252 (1993), 1 $-23$.

[5] Avantaggiati, A.: Teoremi di immersione negli spazi di Sobolev-Besicovitch. Preprint. Rome: Dept. Met. Mod. Mat. 1995, pp. 1-30.

[6] Avantaggiati, A.: Teoria "debole" delle funzioni quasi periodiche. In: Atti del Convegno in Onore di Carlo Ciliberto, Napoli (Italy), 25-26 Maggio 1995 (eds.: T. Bruno et al.). Napoli: La Citta' del Sole 1997, pp. 47 - 90.

[7] Bersani, A. M.: On the Hausdorff-Young theorem for Hilbert vector valued almost periodic functions of Besicovitch spaces. Rend. Mat. (Serie VII) 16 (1996), 143 - 152.

[8] Besicovitch, A. S.: Almost Periodic Functions. Cambridge: Univ. Press 1932.

[9] Dell'Acqua, G. and P. Santucci: Embedding theorems for Sobolev-Besicovitch spaces $W_{a p}^{m, 1}$. Rend. Mat. (Serie VII) 16 (1996), 525 - 536.

[10] Nikišin, E. M.: Dirichlet series with independent exponents and some of their applications. Math. USSR Sbornik 25 (1975), 1 - 36.

[11] Pankov, A. A.: Bounded and Almost Periodic Solutions of Nonlinear Operator Differential Equations. London: Kluwer Acad. Publ. 1990.

[12] Shubin, M. A.: Almost periodic functions and partial differential operators. Russian Math. Surveys 33 (1978), 1 - 52.

[13] Stein, E. M. and G. Weiss: Introduction to Fourier Analysis on Euclidean Spaces. Princeton: Univ. Press 1971.

[14] Zygmund, A.: Trigonometric Series. Cambridge: Univ. Press 1993.

Received 16.12.1996; in revised form 17.02.1998 